\title{
Formability of Thin-Walled Commercial Pure Titanium Tube upon Rotary Draw Bending
}

\author{
Li Guangjun ${ }^{1}, \quad$ Yang Heng ${ }^{2}, \quad$ Xu Xudong ${ }^{1}, \quad$ Li Heng ${ }^{2}, \quad$ Yang He $^{2}$ \\ ${ }^{1}$ Chengdu Aircraft Industry (Group) Corporation Ltd., Chengdu 610092, China; ${ }^{2}$ State Key Laboratory of Solidification Processing, \\ Northwestern Polytechnical University, Xi'an 710072, China
}

\begin{abstract}
Under variations of bending radii and material properties, the multi-defect constrained formability of thin-walled commercial pure titanium (CP-Ti) tube was identified to explore the forming potential of this advanced lightweight material via explicit/implicit 3D-FE models and the experiments. The major results show that the variations of hardening exponents, thickness anisotropy exponent and Young's modulus, have great effects on bending formability; and compared with the wall thinning and cross-section deformation, the wrinkling instability is the dominant defect to restrain the bending formability of the large diameter thin-walled (LDTW) CP-Ti tube. The bending formability of CP-Ti tube, especially the anti-wrinkling capability, can be improved at elevated temperatures.
\end{abstract}

Key words: bending; commercial pure titanium tube; formability; material properties; defect

In aircraft, the potential usage of thin-walled $(D / t>20$, $D$-diameter, $t$-wall thickness) CP-Ti bent tube in hydraulic, fuel and environmental control systems may improve the overall performance of plane by replacing the conventional stainless steel ones ${ }^{[1]}$. The rotary-draw-bending (RDB) is the most universal one to achieve the precision and efficient bending deformation at tight bending conditions by strict controlling of the multiple tools. Currently, the large diameter $(D>40)$ thin-walled CP-Ti tube under small bending radii is designed to further enhance the function performance. However, due to uneven bending deformation caused by extreme geometrical dimensions ${ }^{[2]}$, the multiple undesired phenomena may occur easily such as wrinkling, over-thinning and cross-section distortion. Also, due to unique property of the Alpha CP-Ti with hexagonal close-packed (hcp) structure, the multiple instabilities may prone to occur, especially, the significant springback occurs because of large deformation resist force and small Young's modulus ${ }^{[3]}$. It is noted that, the CP-Ti tube is fabricated by the multi-pass thermal-mechanical processing such as extrusion, rolling, welding and annealing.
Thus, the variations of Ti-tube properties become more significant even with the same batch. Therefore, the bending formability of LDTW CP-Ti tube should be identified under tough bending conditions, viz., small bending radii and obvious property variations.

Great efforts have been done on bending behaviors of different tube bending processes. Wang et al. ${ }^{[4]}$ numerically studied the effects of internal pressure and tube end constraint on wrinkling tendency in hydro-bending of thin-walled Al-alloy tube. Hasanpour et al. ${ }^{[5]}$ numerically studied the effect of anisotropy on ovalization, thickness and wrinkling of tube in $\mathrm{RDB}$, and found that the larger thickness anisotropy index leads to the higher ovalization ratio and smaller wrinkling tendency. Mentella and Strano ${ }^{[6]}$ obtained the correlation between the main geometrical tube parameters of the RDB process and the cross-section deformation related bending quality of the small diameter hydraulic pipings. Wang et al. ${ }^{[7]}$ studied the influence of the springback in high strength TA18 tubes on the wall thinning and section distortion under different process parameters. Daxin et al. ${ }^{[8]}$ derived the formulae to calculate the

$\overline{\text { Received date: January 14, }} 2017$

Foundation item: National Natural Science Foundation of China (50905144); NPU Foundation for Fundamental Research (JC201028); National Science Fund for Excellent Young Scholars (51522509)

Corresponding author: Li Guangjun, Master, Senior Engineer, Chengdu Aircraft Industry (Group) Corporation Ltd, Chengdu 610092, P. R. China, Tel: 0086-28-87405742, E-mail: lgjwcl@sina.com 
springback angles of tubes subjected to combined bending and additional tension during RDB. Recently, Jiang et al. ${ }^{[9]}$ numerically studied the coupling effects of material properties and bending angle on the springback angle of medium-strength Ti-3Al-2.5V tube during RDB. However, there is a lack of research on bending formability of the CP-Ti tube.

Thus, the bending formability of CP-Ti tube upon RDB is addressed via the experimental and numerical methods. First, the variations of CP-Ti tube mechanical properties are obtained by the uniaxial tension test. Then, the explicit/implicit 3D-FE models of RDB for LDTW CP-Ti tube are developed and validated by the experiments. Thus, the bending formability of CP-Ti tube upon cold RDB is explored, and the bending behaviors under variations of mechanical properties are further identified. The bending of CP-Ti tube at elevated temperature is finally conducted to further improve the bending formability of this larger diameter thin-walled CP-Ti tube.

\section{Mechanical Properties of CP-Ti Tube}

According to GB/T 228-2002, the uniaxial tension test of the curved CP-Ti sample was conducted under the tension velocity of $12 \mathrm{~mm} / \mathrm{min}^{[10]}$. The properties of the parent tube are experimentally presented and implemented into the FE model. The true stress-strain curve is fitted to the exponent function of $\bar{\sigma}=K(a+\bar{\varepsilon})^{n}$.

Fig. 1 shows the variation range of the strain hardening exponent $n$ and Young's modulus $E$ under different geome-
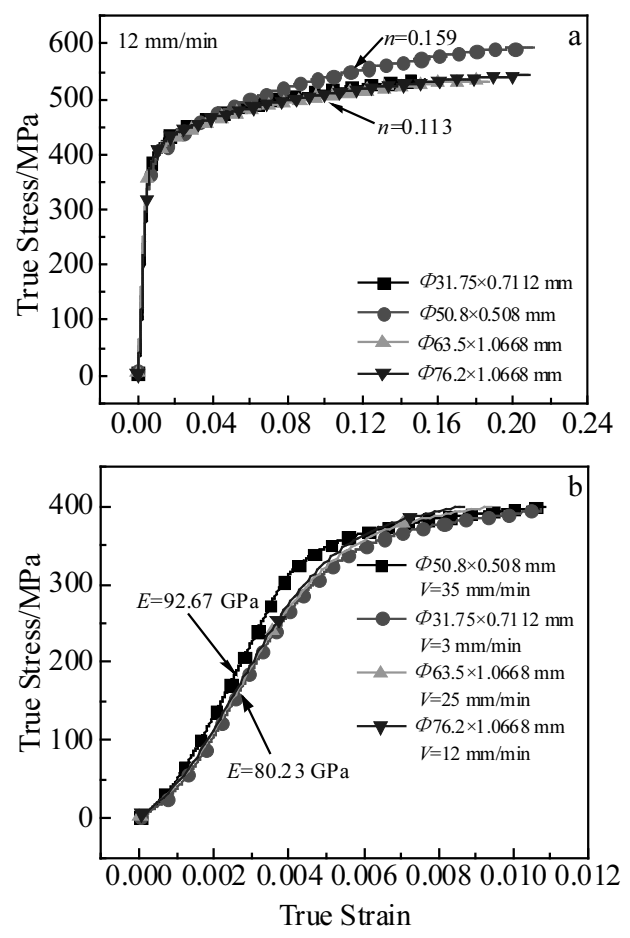

Fig. 1 Variations of $n$ and $E$ of CP-Ti tube with different tension velocities and tube diameters trical dimensions. It is found that the hardening exponent ranges from 0.113 to 0.159 , and Young's modulus ranges from $80.23 \mathrm{GPa}$ to $92.67 \mathrm{GPa}$. Meanwhile, by the calculation of experimental results, it is found that the thickness anisotropy exponent $r$ ranges from 1.3 to 1.7 . It is concluded that the tubes with different geometrical dimensions possess the fluctuation of the mechanical properties. Thus, the obvious variations of CP-Ti tube properties should be concerned in bending formability study.

\section{3D FE Models}

\subsection{D-FE models}

Under FE platform ABAQUS, a series of elastic-plastic 3D-FE models are established as shown in Fig. 2 to simulate RDB of LDTW CP-Ti tube under different bending radii and variation of mechanical properties.

The Hill's anisotropic quadratic yield function with isotropic hardening is used to describe tube material's yield behaviors. Since relative diameter $D / t$ equals 100 , the shell element $S 4 \mathrm{R}$ is used to model the tube with the plane stress conditions. The rigid tools are modeled with four-node bilinear quadrilateral rigid element R3D4. The explicit algorithm is used for tube bending and balls retracting; while the implicit one is used for unloading process (springback) ${ }^{[11,12]}$. The boundary constraints are applied by two approaches to realize the actual process of RDB: 'displacement/rotates' and 'velocity/angular'. The trapezoidal profile is used to define the smooth loading of the tools to reduce inertial effects in explicit simulation of the quasi-static process. The 'surface-to-surface method' is used to define the contact behaviors between tube and various dies, which allows finite sliding between the above surfaces except for tube-clamp die interface.

\subsection{Evaluation of 3D-FE model}

The experiments with the bending radius of $2 \mathrm{D}$ and $3 \mathrm{D}$ are conducted to verify the 3D-FE model. The forming conditions can be found in Table 1. It is noted that the mandrel diameter is $49.64 \mathrm{~mm}$, viz., the clearance of tube-mandrel is $0.144 \mathrm{~mm}$.

First, for bending case of 2D, the clearance between tube and wiper die is assigned as $0.1 \mathrm{~mm}$ to induce the wrinkling. Fig. 3

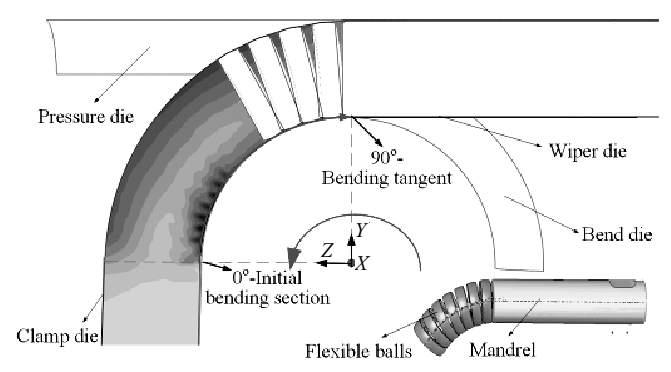

Fig. 2 3D-FE model of CP-Ti tube RDB with multi-die constraints under bending radius of $2 \mathrm{D}$ 
Table 1 Forming parameters for different bending specifications

\begin{tabular}{cc}
\hline Parameters & Value \\
\hline Relative pushing speed, $V_{\mathrm{p}} / V / \%$ & 100.0 \\
Bending angle $/ \mathrm{rad}$ & $\pi / 2$ \\
Mandrel diameter, $d / \mathrm{mm}$ & 49.64 \\
Mandrel extension length $/ \mathrm{mm}$ & $0 \sim 6$ \\
Thickness of balls $/ \mathrm{mm}$ & 12.5 \\
Pitch of balls $/ \mathrm{mm}$ & 2.0 \\
Number of balls & $2 \sim 6$ \\
Ball diameter $/ \mathrm{mm}$ & 49.54 \\
Length of clamp die $/ \mathrm{mm}$ & 203 \\
\hline
\end{tabular}

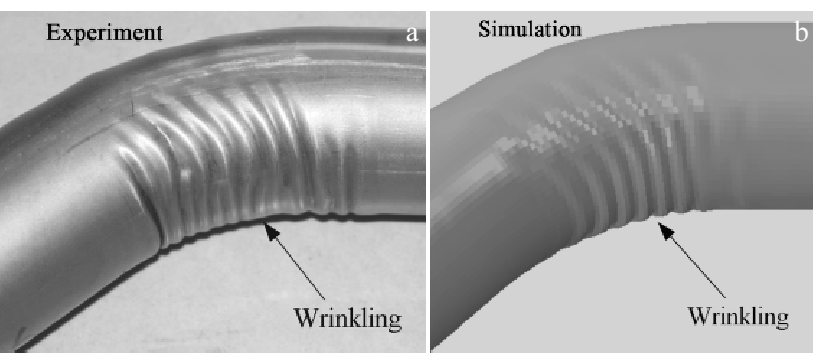

Fig.3 Comparisons of wrinkling between experimental (a) and simulation (b) results

shows the comparison of bent tubes between experimental and simulation results. We observe that the wave height of 3D-FE prediction is similar to the experimental one. Second, the bending behaviors under bending radius of $2 \mathrm{D}$ and $3 \mathrm{D}$ are compared in terms of wall changing and cross-section deformation.

Fig.4a shows that the wall thickening degree fluctuates near the clamp die at the bending radius of $2 \mathrm{D}$, which indicates the minor wrinkling, just as the result of the experiment. It also shows that the numerical results of wall thinning degree are larger than the experimental ones, and the largest relative error is $13.29 \%$. Fig. 4 b shows that, under the bending radius of $3 \mathrm{D}$, the numerical wall thickness changing degree agrees with the results of the experiments with the maximum relative error of $6.61 \%$. For springback angle, it is found that the numerical prediction for $2 \mathrm{D}$ and $3 \mathrm{D}$ is $2.42^{\circ}$ and $4.12^{\circ}$, respectively. The experimentally measured ones are $2.47^{\circ}$ and $3.77^{\circ}$. Thus, we think that the 3D-FE models are reliable.

\section{Results and Discussion}

\subsection{Cold bending formability under various bending radii}

Via the verified 3D-FE models, the cold-bending formability of thin-walled CP-Ti tube upon RDB is explored under different forming conditions, viz., various bending radii and variations of mechanical properties. The number of balls, mandrel extension length and the clearance between tube and mandrel, used in the study, are 4, 3 and $0.14 \mathrm{~mm}$, respectively. The bending radius of CP-Ti tube consist of 1.0D, 1.5D, 2.0D, 2.5D and 3.0D.
Fig.5 and Fig. 6 show the changing tendency of wall thickness changing degree, cross-section ovalization degree
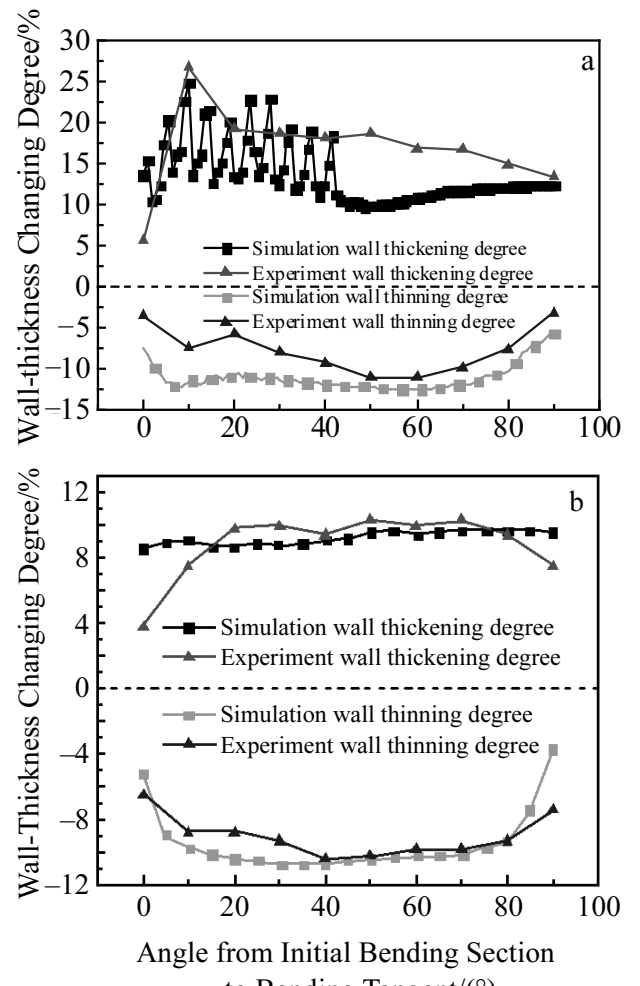

to Bending Tangent $/\left(^{\circ}\right)$

Fig.4 Comparisons of the wall thickness changing degrees between experiment and simulation: (a) 2D and (b) 3D
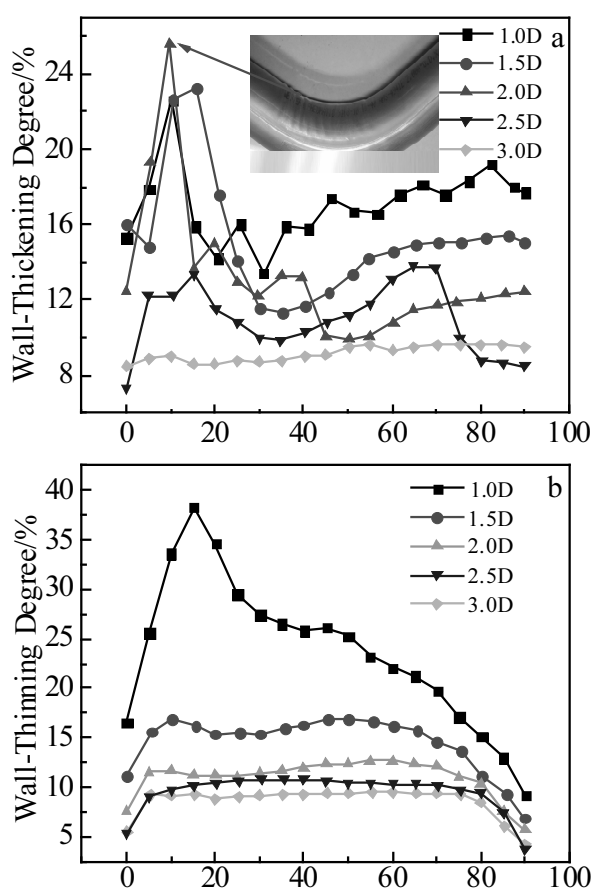

Angles from Initial Bending Section to Bending Tangent $/\left(^{\circ}\right)$

Fig. 5 Wall thickness changing of CP-Ti tube under different bending radii 
and springback with the changing of bending radii. From Fig.5a, we found that the fluctuation degrees of wall thickening increases with the decreasing of the bending radius, which indicates the increased wrinkling tendency. Fig.5b and Fig.6 show that both wall thinning degree and cross-section ovalization increase with the decreasing of the bending radius. The smaller the bending radius, the larger the increasing tendency. It is noted that, for bending of $1.0 \mathrm{D}$ case, the wall-thinning degree is nearly $40 \%$, and the cross-section ovalization is serious, viz., up to $10 \%$. It is also observed that springback angle increases with the increasing of the bending radius. This is because the regions involved in the deformation increases with larger bending radius, which results in the larger elastic deformation at the deformed zone.

From the above analysis, we concluded that the bending deformation of thin-walled CP-Ti tube tends to deteriorate with the decreasing of the bending radius. The bending radius should be larger than $2.0 \mathrm{D}$ to reduce the wrinkling tendency, and the bending radius should larger than $1.0 \mathrm{D}$ to avoid the over thinning and cross-section ovalization. Thus, to get the stable deformation of LDTW CP-Ti tube at room temperature, the bending radius should be larger than 2.0D. The qualified bent CP-Ti tube with the bending radius of $3 \mathrm{D}$ is obtained without wrinkling, and the maximum wall thinning is $10.28 \%$, the cross-section ovalization less than 3\%. Meanwhile, by adjusting the processing parameters, the qualified bent part with 2D is achieved with the maximum wall thinning of the qualified part of $11.43 \%$, cross-section ovalization of $2.69 \%$ and wrinkling height less than $2 \%$.

\subsection{With variations of material properties}

\subsubsection{Under variation of Young's modulus $E$}

The property variations related bendability is addressed under two bending radii of $2 \mathrm{D}$ and 3D. Fig.7 shows that Young's modulus has little effect on the change of wall thickness, and the prediction results show that it also has neglected effects on wrinkling tendency.

Fig. 8 shows that the cross-section ovalization tends to decrease with the increasing of the Young's modulus. This is

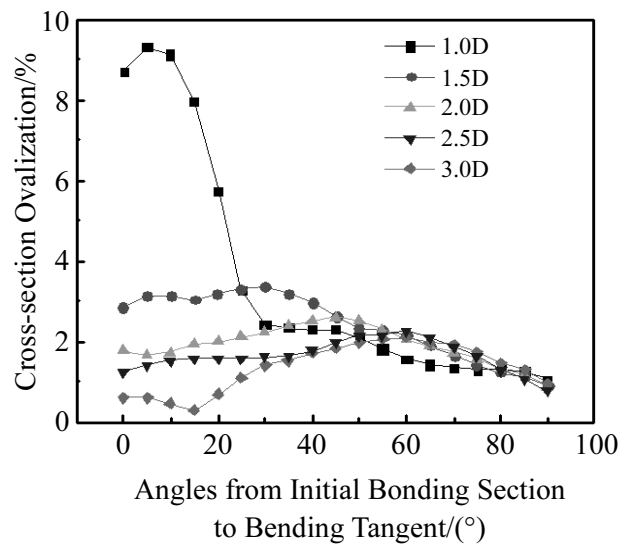

Fig. 6 Cross-section ovalization of CP-Ti tube with different bending radii

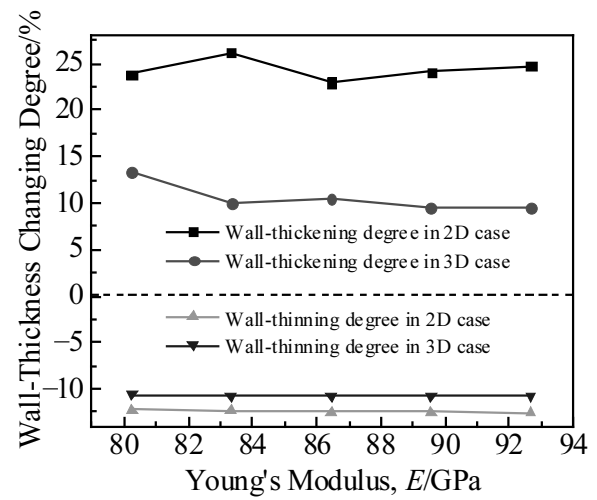

Fig.7 Wall thickness changing under different Young's modulus

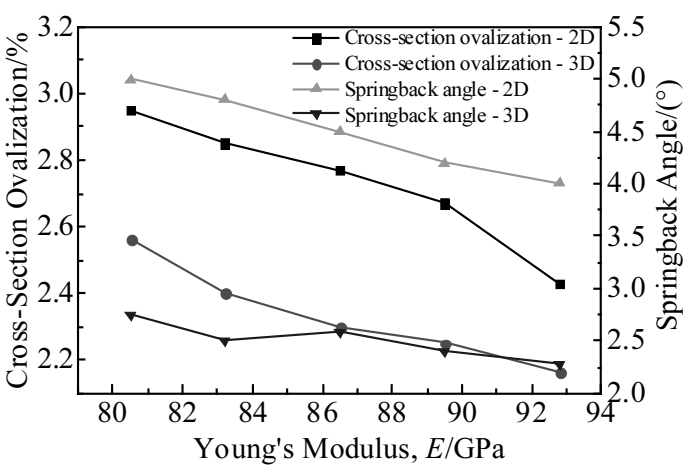

Fig. 8 Cross-section ovalization and springback angle under different Young's modulus

because the elastic modulus represents the characteristic of material stiffness, and the stiffness increases with the increasing of Young's modulus, which strengthens the ability of the CP-Ti tube to resist cross-section distortion. In addition, Fig. 8 shows that the springback angle also decreases with the increasing of the elastic modulus. The reason is that the greater springback occurs for the CP-Ti tube with smaller $E$.

3.2.2 Under variation of strain hardening exponent

Fig. 9 shows the prediction bent tubes under different values of strain hardening exponent $n$ for different bending radii. We observe that the wrinkling occurs for bending radii of $2 \mathrm{D}$, while the wrinkling with $n$ of 0.11 is more serious than that with $n$ of 0.16 . However, the effect of strain hardening exponent is not obvious at the bending radius of 3D. Fig.10 shows the wall thickness changing tendencies of thin-walled CP-Ti tube with different $n$ under the bending radii of $2 \mathrm{D}$ and 3D. It is evident that the wall thickness changing degree decreases greatly with the increasing of $n$ values, and this phenomenon becomes much more obvious under bending radius of 2D. It implies that the effect significance of strain hardening exponent increases when the tube is subjected to larger plastic deformation under small bending radii. 


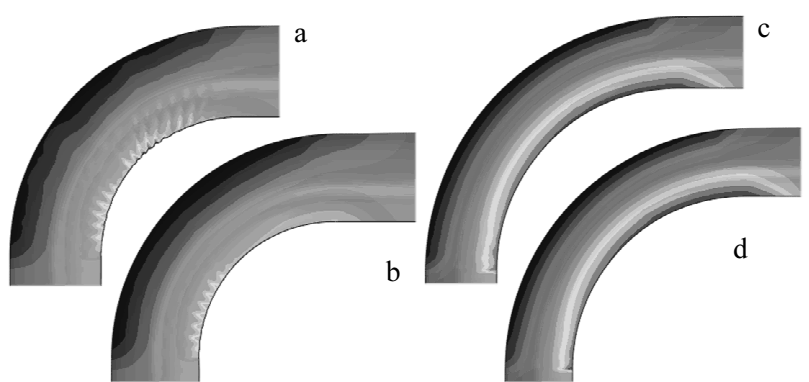

Fig.9 Simulation results under different strain hardening exponents: (a) $n=0.11,2 \mathrm{D}$; (b) $n=0.16,2 \mathrm{D}$; (c) $n=0.11,3 \mathrm{D}$; (d) $n=0.16,3 \mathrm{D}$

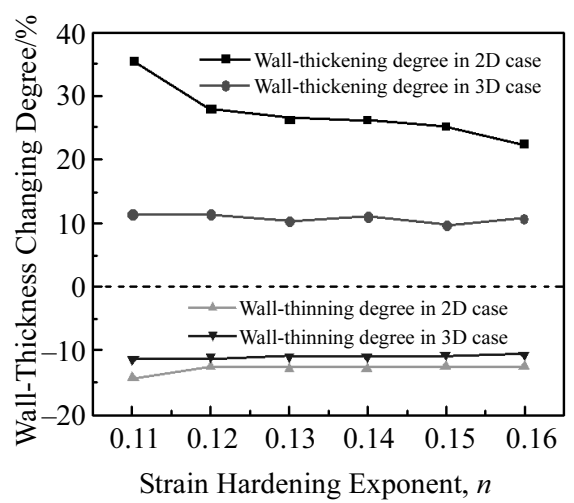

Fig.10 Wall thickness changing under different strain hardening exponents

Fig.11 shows that the strain hardening exponent has little effect on the cross-section ovalization except when $0.11<n<0.12$ at $2 \mathrm{D}$ case. The reason is that the serious wrinkling occurs when $n=0.11$ at $2 \mathrm{D}$, which causes abnormal cross-section ovalization. Fig. 11 also shows that, under different bending radii, the effects of the strain hardening exponent $n$ are different from each other. Under the bending radius of $3 \mathrm{D}$, the springback angle decreases with the increasing of the strain hardening exponent $n$, while the opposite tendency is observed with the bending radius of 2D. The reason is that, within small strain ranges, the amount of elastic deformation decreases with the larger strain hardening exponent. Since the bending deformation is relatively small, it can be concluded that the small elastic deformation results in small springback of thin-walled CP-Ti tube upon bending. However, why the contrary tendency happens under 2D case is attributed to the occurring of the wrinkling instability. Under small bending radii, the increasing tendency of springback is mainly caused by the decreased wrinkling tendency with the increasing of strain hardening exponent. When the wrinkling occurs, the elastic deformation can recover at the wrinkling zone, which reduces the elastic recovery after bending and leads to the smaller springback angle.

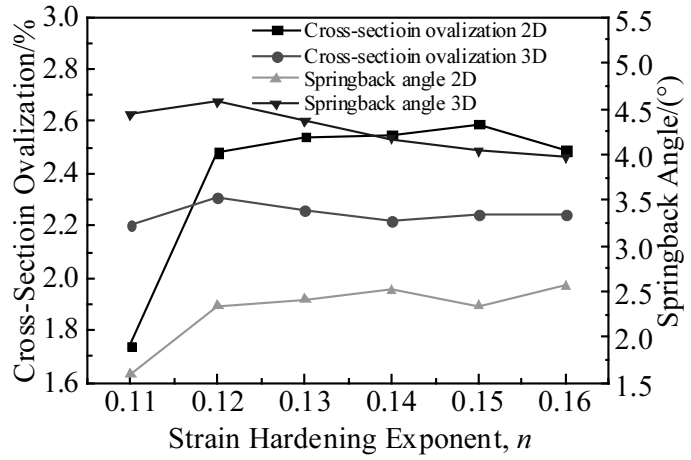

Fig. 11 Cross-section ovalization and springback angle under different strain hardening exponents

\subsubsection{Under variation of thickness anisotropy exponent $r$}

Fig.12 shows the wall thickness changing tendency with the thickness anisotropy exponent $r$. We found that with the increase of $r$, the wall thinning degree decreases for both bending radii. While the wall thickening degree varies little especially under the bending radius of $3 \mathrm{D}$. Since the minor wrinkling occurs under the bending radius of $2 \mathrm{D}$, the changing of $r$ value has limited effects on reducing the fluctuations of the wall thickness at the inner side of the tube, which leads to the unobvious changing of the wall thickening degree.

Fig.13 shows the cross-section ovalization and springback angle of the thin-walled CP-Ti tube with different thickness anisotropy exponent $r$. It can be seen that both the cross-section ovalization and springback angle tend to decrease with the increasing of the thickness anisotropy exponent. Due to the decreased wall thinning degree of the CP-Ti tube with increasing $r$, the capability of bent tube to resist the cross-section ovalization increases. Meanwhile, because of the decreased cross-section ovalization and larger plastic deformation, the springback angle decreases after unloading.

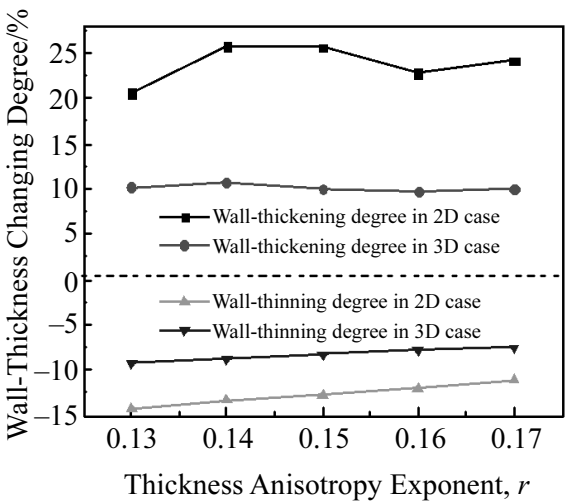

Fig. 12 Wall thickness change degree under different thickness anisotropy exponents 


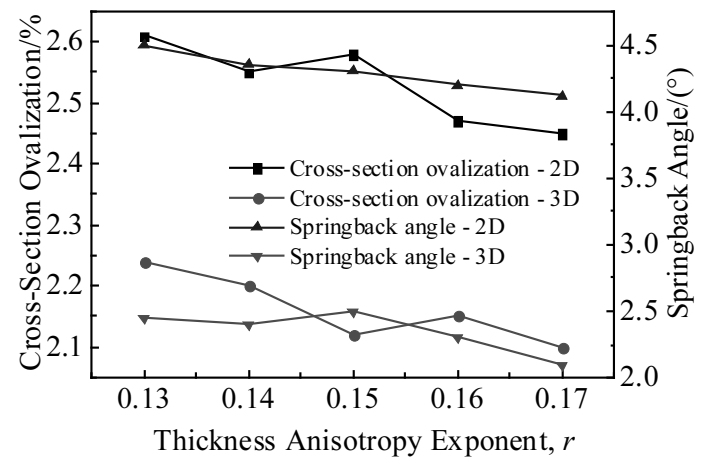

Fig. 13 Cross-section ovalization and springback angle under different thickness anisotropy exponents

\subsection{Improved bending formability at elevated temperatures}

From the above analysis, we found that the wrinkling instability is the dominant defect to restrain the bending limit and bending quality of LDTW CP-Ti tube upon cold-bending. To achieve the smaller bending radius less than $2 \mathrm{D}$, the bendability of the CP-Ti tube at elevated temperatures is studied. Based on the ABAQUS/Explicit module, using the coupled thermo-mechanical dynamic explicit algorithm, a 3D coupled thermo-mechanical FE model is established for LDTW CP-Ti tube bending at elevated temperatures. In the models, the mandrel and pressure die are heated, and the heat is then transferred to the tube so that the tube can obtain a preset temperature field.

For the bending radius of $1.5 \mathrm{D}$, the formability of CP-Ti tube upon bending at elevated temperatures is numerically studied. Fig. 14 shows the comparison of the CP-Ti bent tube upon cold bending with heat bending. It can be seen that the wrinkling instability of the CP-Ti tube is avoided at elevated temperatures. Fig. 15 further confirms the positive role of elevated temperature on anti-wrinkling tendency. It is found that the wrinkling free bent tubes with the bending radius of $1.5 \mathrm{D}$ can be achieved with the temperature ranging from $300{ }^{\circ} \mathrm{C}$ to $400{ }^{\circ} \mathrm{C}$.

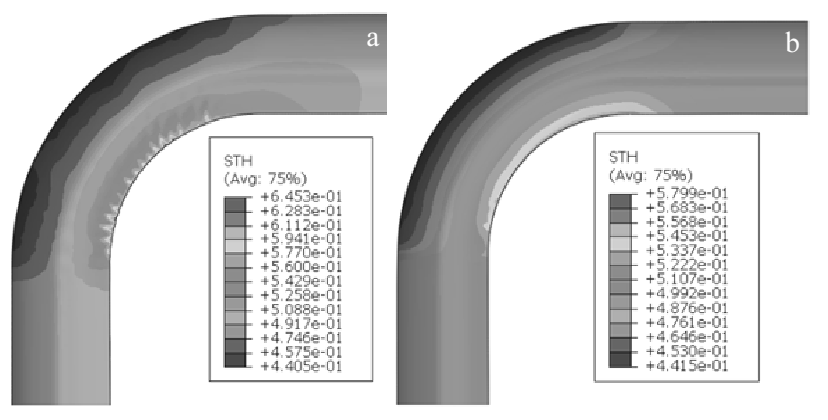

Fig. 14 Comparison of the CP-Ti bent tube using cold bending method (a) and heat bending method (b)

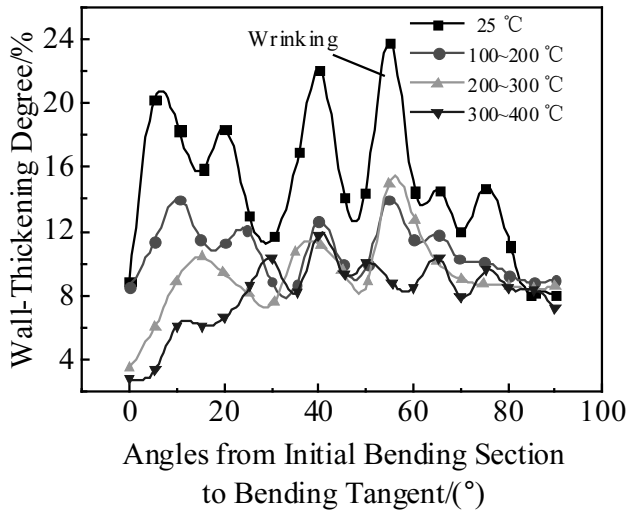

Fig. 15 Wall thickening degree at different heating temperatures using heat bending method

\section{Conclusions}

1) There occur obvious variations of mechanical properties of CP-Ti tube regarding Young's modulus, strain hardening exponent and thickness anisotropy exponent. The strain hardening exponent has a relatively larger impact on wrinkling tendency; With the increasing of the thickness anisotropy exponent, the wall thinning degree decreases; The Young's modulus has great effects on both cross-section deformation degree and springback.

2) The bending radius should be larger than $2.0 \mathrm{D}$ to avoid the wrinkling occurring for LDTW CP-Ti tube upon cold bending, while the bending radius should be larger than 1.0D to avoid the over thinning and cross-section distortion. Wrinkling is the dominant defect to restrain the bending limit of LDTW CP-Ti tube upon cold-bending. Considering the positive effect of material properties on wrinkling restraining, the bending at elevated temperatures is used to achieve the tough bending conditions with smaller bending radius less than 2.0D.

\section{References}

1 Schweibold D J. Aircraft Engineering \& Aerospace Technology [J], 1989, 61(12): 8

2 Yang $\mathrm{He}$, Li He, Zhang Zhiyong et al. Chinese Journal of Aeronautics[J], 2012, 25(1): 1

3 Boyer R R, Welsch G, Collings E W. Materials Properties Handbook: Titanium Alloys[M]. Materials Park, OH: ASM International, 1994

4 Wang Xiaosong, Song Peng, Zhao Zhongyuan et al. Transactions of Nonferrous Metals Society of China[J], 2011, 21: 440

5 Hasanpour K, Amini B, Poursina $\mathrm{M}$ et al. AIP Conference Proceedings[J], 2011, 1383: 187

6 Mentella A, Strano M. Proceedings of the Institution of Mechanical Engineers Part B Journal of Engineering Manufacture[J], 2012, 226: 267

7 Wang Yan, Yang He, Li Heng et al. Rare Metal Materials and 
Engineering[J], 2012, 41(7): 1221

8 Daxin E, Guan Zhiping, Chen Jisheng. Journal of Materials Engineering \& Performance [J], 2012, 21(11): 2316

9 Jiang Zhiqiang, Yang He, Zhan Mei et al. Materials \& Design[J], 2010, 31: 2001
10 AMS4941E[S]. America: ASM International, 2008

11 Li Heng, Yang He, Song Feifei et al. Rare Metal Materials and Engineering[J], 2014, 43(1): 64

12 Song Feifei, Yang He, Li Heng et al. Rare Metal Materials and Engineering[J], 2013, 42(1): 43

\section{薄壁纯钛管数控弯曲成形性能研究}

李光俊 ${ }^{1}$, 杨 恒 $^{2}$, 许旭东 ${ }^{1}$, 李 恒 $^{2}$, 杨 合 $^{2}$

(1. 成都飞机工业(集团)有限责任公司, 四川 成都 610092)

(2. 西北工业大学 凝固技术国家重点实验室, 陕西 西安 710072)

摘 要: 研究了薄壁纯钛 ( CP-Ti) 管在不同弯曲半径和不同材料性能下的多缺陷约束数控弯曲成形性能, 通过显式/隐式三维有限元模 型和实验研究了先进轻量化材料 CP-Ti 的成形潜力。结果表明: 管材的硬化指数、厚向异性指数和杨氏模量的变化对弯曲成形性能有很 大影响; 与壁厚减薄和截面畸变相比, 失稳起皱是抑制大直径薄壁 $\mathrm{CP}-\mathrm{Ti}$ 管弯曲成形性能的主要缺陷。升高温度可以提高 $\mathrm{CP}-\mathrm{Ti}$ 管的弯 曲成形性能, 特别是抗起皱性能。

关键词: 弯曲; 商业纯钛管; 成形性能; 材料性能; 缺陷

作者简介: 李光俊, 男, 1970 年生, 硕士, 高级工程师, 成都飞机工业(集团)有限责任公司, 四川 成都 610092, 电话: 028-87405742, E-mail: lgjwcl@sina.com 